\title{
Phagocytes transmit Chlamydia pneumoniae from the lungs to the vasculature
}

\author{
J. Gieffers*, G. van Zandbergen*, J. Rupp*, F. Sayk", S. Krüger", S. Ehlers ${ }^{\star}$, W. Solbach*, M. Maass*
}

Phagocytes transmit Chlamydia pneumoniae from the lungs to the vasculature. J. Gieffers, G. van Zandbergen, J. Rupp, F. Sayk, S. Krüger, S. Ehlers, W. Solbach, M. Maass. (C) ERS Journals Ltd 2004.

ABSTRACT: Chlamydia pneumoniae, a major cause of community-acquired pneumonia, primarily infects the respiratory tract. Chronic infection of nonrespiratory sites, such as the vascular wall, the brain or blood monocytes, requires evasion from the lungs and spreading via the bloodstream. The cell types involved in dissemination are insufficiently characterised.

In this study, New Zealand White rabbits were infected intratracheally with $C$. pneumoniae, and lung manifestation and systemic dissemination were monitored by polymerase chain reaction and immunohistochemistry. Infection of the lungs was characterised by an early phase dominated by granulocytes and a late phase dominated by alveolar macrophages (AM).

Granulocytes, AM and alveolar epithelial cells acted as host cells for chlamydiae, which remained detectable for up to 8 weeks. AM transported the pathogen to the peribronchiolar lymphatic tissue, and subsequently $C$. pneumoniae entered the spleen and the aorta via dissemination by peripheral blood monocytes.

In conclusion, Chlamydia pneumoniae-infected alveolar macrophages transmigrate through the mucosal barrier, and give the pathogen access to the lymphatic system and the systemic circulation. Infected peripheral blood monocytes are the vector system within the bloodstream and transmit the infection to the vascular wall. This is the first description of granulocytes acting as a reservoir for Chlamydia pneumoniae early in infection.

Eur Respir J 2004; 23: 506-510.
*Institute for Medical Microbiology and Hygiene, ${ }^{\#}$ Institute for Pathology, University of Lübeck, Lübeck and Research Center Borstel, Division of Molecular Infection Biology, Borstel, Germany.

Correspondence: J. Gieffers, Institute for Medical Microbiology and Hygiene, University of Lübeck, Ratzeburger Allee 160, 23538 Lübeck, Germany.

Fax: 494515002808

E-mail: jens.gieffers@hygiene.ukl.mu-luebeck.de

Keywords: Animal model

chlamydia

dissemination

macrophages

monocytes

vascular infection

Received: August 132003

Accepted: November 252003

This study was supported by a grant from the Deutsche Forschungsgemeinschaft to M. Maass (SFB 367, B11).
Chlamydia pneumoniae has been established as the third species of the genus chlamydia and has emerged as the cause of $\sim 10 \%$ of community-acquired pneumonias, and $5 \%$ of pharyngitis and sinusitis cases [1]. The seroprevalence in the adult population is as high as $80 \%$, indicating that nearly everybody acquires the infection at least once during their lifetime and that re-infections often occur [2]. C. pneumoniae has been associated with several nonrespiratory diseases, such as multiple sclerosis [3], Alzheimer's disease [4] or coronary heart disease [5]. As no chlamydiae elementary bodies have ever been detected circulating freely throughout the bloodstream, it must be assumed that a cellular vector system transports the obligate intracellular pathogen from the respiratory origin of infection to the vascular wall. Within the bloodstream, monocytes have been shown to harbour viable chlamydiae in vivo $[6,7]$ and to transmit the pathogen to endothelial or smooth muscle cells in vitro [8]. In these cells, chlamydiae enter a persistent infection that is refractory to antimicrobial treatment in vivo [7]. However, the vector cell, which allows the respiratory pathogen to evade from the lungs, has yet to be identified. Previous animal models have suggested macrophages as possible carrier cells [9]. To further investigate this hypothesis, New Zealand White rabbits were inoculated intratracheally, the cell types infected by $C$.

For editorial comments see page 499. pneumoniae during the pulmonary manifestation were identified and the dissemination from the lungs to the systemic circulation was followed by immunohistochemistry (IHC) and polymerase chain reaction (PCR).

\section{Materials and methods}

\section{Cultivation and purification of Chlamydia pneumoniae}

The C. pneumoniae strain CV-6, which was used in this study, was previously isolated from a coronary artery plaque and continuously propagated on HEp-2 cells, as described previously [10]. After confluence of the cell sheet, the growth medium, consisting of Eagle's minimal essential medium (Gibco, Eggenstein, Germany), 10\% foetal bovine serum (Biochrom, Berlin, Germany) and nonessential amino acids (Gibco), was replaced by serum-free Eagle's minimal essential medium supplemented with $1 \mu \mathrm{g} \cdot \mathrm{mL}^{-1}$ cycloheximide (Sigma, Deisenhofen, Germany) and centrifuged at $3,000 \times g$ for $45 \mathrm{~min}$. After incubation at $35^{\circ} \mathrm{C}$ and $5 \%$ carbon dioxide for $72 \mathrm{~h}$, the infected monolayers were mechanically disrupted. Host debris was removed by centrifugation at $1,000 \times g$ for $10 \mathrm{~min}$ and chlamydiae were concentrated by ultracentrifugation of the supernatant at $24,000 \times g$ for $1 \mathrm{~h}$. Inclusion 
forming units (IFU) were determined by inoculating fresh monolayers and staining with a chlamydia-specific, antilipopolysaccharide (LPS) monoclonal fluorescein isothiocyanate (FITC)-coupled antibody (Dako, Ely, England) after 3 days. Mock-infected cells were prepared in the same way and used for inoculation of negative controls.

\section{Experimental animals}

A total of 21 female New Zealand White rabbits, including three negative control animals (1,600-2,000 g; Charles River, Kisslegg, Germany), raised and kept under specific pathogen-free conditions, and fed standard rabbit chow, were anaesthetised with a cocktail of atropine (Fresenius, Homburg, Germany), diazepam (Ratiopharm, Ulm/Donautal, Germany), ketamine (Parke-Davis, Berlin, Germany) and rompun (Bayer, Leverkusen, Germany). Animals were inoculated intratracheally in a nontraumatic fashion with $1 \times 10^{6}$ $0.5 \times 10^{7}$ IFU $C$. pneumoniae CV-6 in $0.5 \mathrm{~mL}$ phosphatebuffered saline through an 8-charière (2.4-mm) catheter, inserted into the trachea, distal to the thyroid cartilage. Twelve animals were inoculated once and sacrificed in batches of two after 12 h, 1 day, 3 days, 7 days, 14 days and 8 weeks. Six animals were inoculated three times at day 0,3 and 7 , and sacrificed in batches of two at 2 weeks, 4 weeks or 8 weeks after the last inoculation. Three control animals were inoculated three times in the same manner with a preparation of mock-infected cells and sacrificed after 3 days, 2 weeks and 4 weeks. Rabbits were sacrificed by intravenous injection of T-61 (Hoechst Roussel Vet, Unterschleissheim, Germany). Lungs, spleen and aorta were removed from the animals. Only single-use instruments were used to prevent cross contamination. One part of the sample was fixed in $10 \%$ buffered formalin and embedded in paraffin for histological examination and IHC. The other part was homogenised and deoxyribonucleic acid (DNA) was extracted using Qiagen columns (Qiagen, Hilden, Germany), according to the manufacturer's instructions. Peripheral blood monocytes (PBMC) were isolated from the blood using Histopaque (Sigma) gradient centrifugation according to standard protocols.

\section{Polymerase chain reaction}

The PCR assay used was consistent with Centers for Diseases Control and Prevention (CDC) recommendations for $C$. pneumoniae PCR [11]: $100 \mu \mathrm{g}$ of extracted rabbit DNA was used for a $C$. pneumoniae-specific nested PCR based on the species-specific HL-1/HR-1 primer pair and on the nested IN-1 (5' AGTTGAGCATATTCGTGAGG 3'), IN-2 (5' TTTATTTCCGTGTCGTCCAG $3^{\prime}$ ) primer pair, which yields a 128-base pair product of the pst $\mathrm{I}$ fragment, as previously described in detail [12]. Each PCR step contained 32 cycles of $1.5 \mathrm{~min}$ at $95^{\circ} \mathrm{C}, 1 \mathrm{~min}$ at $55^{\circ} \mathrm{C}$ and $1.75 \mathrm{~min}$ at $72^{\circ} \mathrm{C}$. For confirmation and enhancement of sensitivity and specificity, a nonradioactive DNA hybridisation was performed using the digoxigenin-labelled HM-1 oligonucleotide probe [12]. In order to assess the sensitivity of the PCR protocol, ten-fold dilutions of a plasmid containing the target sequence (pGMP, 3475 base-pairs (Promega, Madison, Wisconsin USA)) were used as a template. The sensitivity of nested PCR and hybridisation ranged 1-100 plasmid copies. To disclose the presence of PCR inhibitors in each sample, $1 \times 10^{2}$ copies of the plasmid were added to the genomic DNA and the nested PCR was repeated as described above.

\section{Immunohistochemistry}

A total of $1004-\mu \mathrm{m}$ thin paraffin sections of each sample were prepared to detect chlamydial antigens immunohistochemically using the avidin-biotin peroxidase-complex method (Vectastain ABC Elite; Vector Laboratories, Burlingame, CA, USA). Forty sections each were stained with the CF-2 genusspecific monoclonal antibody (dilution 1:8,000; Washington Research Foundation, Seattle, WA, USA) and with a monoclonal C. pneumoniae-specific anti-lipopolysaccharide antibody (dilution 1:500; kindly provided by $\mathrm{H}$. Brade, Research Center Borstel, Borstel, Germany) as primary antibody. An isotypeidentical (immunoglobulin (Ig)G2a) monoclonal antibody (anti-human CK 13, DE-K13; Dako, Hamburg, Germany) and mouse ascitic fluid (Sigma) served as negative controls (10 sections each). Consecutive sections of the lungs were stained with a monoclonal anti-rabbit macrophage antibody, RAM 11 (Dako, Germany). A biotinylated rabbit-anti-mouse-IgG (Dako, Germany) was applied as a second antibody, followed by incubation with horseradish peroxidase-labelled streptavidin (Dako, Germany) and detection with diaminobenzidinesolution (Sigma). Finally, slight counterstaining with haematoxylin was performed. Data were interpreted according to the CDC-recommendations for $C$. pneumoniae tissue diagnostics [11]; a sample was considered positive when specific reactivity was observed with both chlamydial antibodies in the absence of reactivity in the negative controls.

\section{In vitro infection of granulocytes with Chlamydia pneumoniae}

Whole blood obtained from healthy adult female New Zealand White rabbits was layered on a Histopaque $\mathbb{R}$ gradient consisting of two layers, Histopaque 1,071 (top) and Histopaque 1,119 (bottom) (Sigma), and centrifuged for $5 \mathrm{~min}$ at $300 \times g$, followed by $15 \mathrm{~min}$ at $800 \times g$ (without brake). The granulocyte-rich layer of Histopaque 1,119 was collected and washed twice in RPMI 1640 medium (Seromed-Biochrom, Berlin, Germany), and the cells were further fractionated on a discontinuous Percoll (Pharmacia, Uppsala, Sweden) gradient, consisting of layers with densities of $1,105 \mathrm{~g} \cdot \mathrm{mL}^{-1}(85 \%)$, $1,100 \mathrm{~g} \cdot \mathrm{mL}^{-1}(80 \%), 1,093 \mathrm{~g} \cdot \mathrm{mL}^{-1}(75 \%), 1,087 \mathrm{~g} \cdot \mathrm{mL}^{-1}(70 \%)$ and $1,081 \mathrm{~g} \cdot \mathrm{mL}^{-1}(65 \%)$. After centrifugation for $25 \mathrm{~min}$ at $800 \times g$, the interface between the 80 and $85 \%$ Percoll layers was collected and washed twice in RPMI 1640. All procedures were carried out at room temperature. Granulocytes were infected with C. pneumoniae elementary bodies, strain CV-6, at a multiplicity of infection of 1 , and cultivated in RPMI 1640 medium (Gibco) supplemented with $2 \mathrm{mM}$ L-glutamine, $10 \mathrm{mM}$ HEPES (all Seromed-Biochrom) and with 10\% foetal calf serum (Gibco) for 4 days at $35^{\circ} \mathrm{C}$. Cytospins were prepared using standard protocols. Cells were fixed with methanol and stained with FITC-conjugated anti-chlamydia LPS monoclonal antibodies (Dako). A total of $1 \times 10^{6}$ infected granulocytes were disrupted by glass beads and passed on one well of a 24 -well cluster plate containing $1 \times 10^{6} \mathrm{HEp}-2$ cells. Chlamydiae were cultivated for two passages as described above. Infected HEp-2 cells were fixed with methanol and stained with a FITC-conjugated anti-chlamydia LPS monoclonal antibody.

\section{Results}

\section{Histopathology}

The $C$. pneumoniae infection of the lungs resulted in an interstitial and alveolar pneumonia with bronchiolitis 
that resolved spontaneously after 2-4 weeks. Repeated inoculations did not result in significantly prolonged disease courses. Histological findings exhibited infiltrates of heterophilic granulocytes and mononuclear cells within the interstitium, alveolar space and bronchiolar lumen. Within $12 \mathrm{~h}$ after infection, an intrabronchiolar and alveolar infiltrate of granulocytes developed. Later, an interstitial infiltrate consisting of granulocytes and a few mononuclear cells was observed from $24 \mathrm{~h}$ to 3 days (fig. 1a). After 3 days, the granulocytic infiltrates within the interstitium, the bronchiolar and alveolar space were replaced by mononuclear cells (fig. 1b). Some animals demonstrated a mild vasculitis and perivasculitis that reached a maximal extent at 3 days post-infection. Perivascular and peribronchiolar lymphatic hyperplasia was observed in all infected animals beginning at day 3 and lasting for the whole period studied. Histological changes were not observed in the spleen and the aorta until the end of the observation period of 8 weeks.

\section{Immunohistochemistry and polymerase chain reaction}

C. pneumoniae was detected by IHC and PCR in the lungs, the spleen, blood monocytes and the aorta, as shown in table 1. C. pneumoniae DNA was present continuously in the lungs for 2 weeks in all animals and for 8 weeks in two out of four animals who had acquired asymptomatic chronic respiratory infection. Antigen was detected up to day 7 after single inoculation and for up to 2 weeks after triple inoculation. Four different cell types were identified within the lungs as target cells of a $C$. pneumoniae infection in vivo. Until day 3, $C$. pneumoniae could not be detected in granulocytes of the lungs by IHC (fig. 1c). Chlamydial antigen was present in alveolar macrophages (AM), alveolar and bronchiolar epithelium during the entire course of infection (fig. 1d, e and f). Infected macrophages were identified in the lymphatic tissue of the lungs, shown by staining of consecutive sections with a macrophage-specific antibody (fig. 1g and h). When circulating PBMC were tested
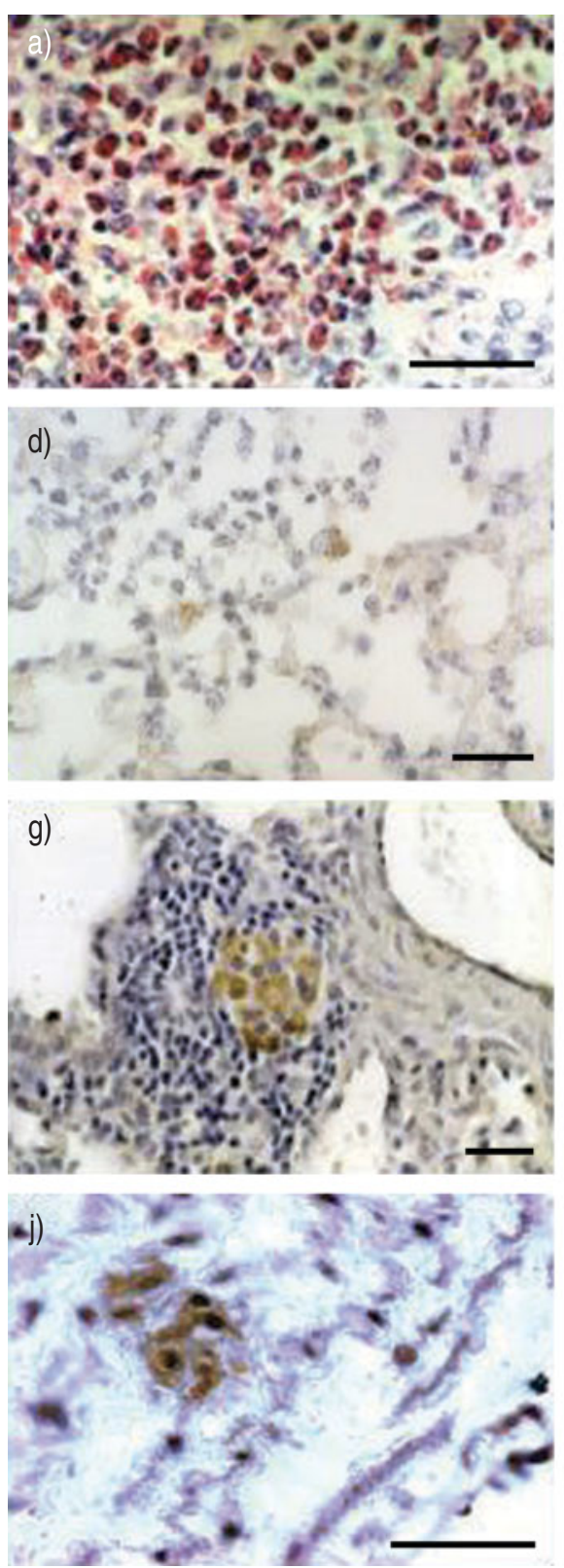
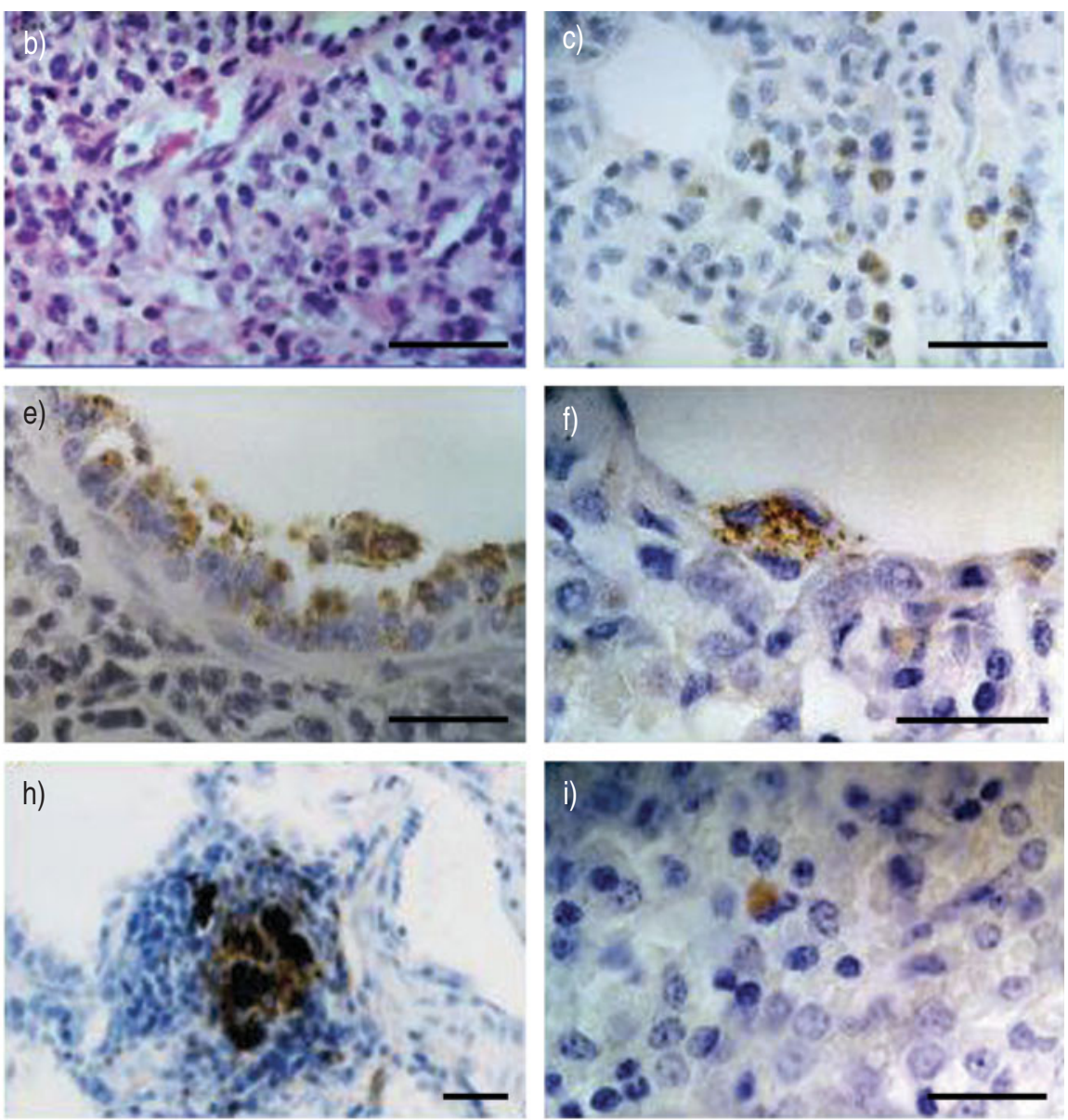

Fig. 1.- Haematoxylin-eosin staining of a Chlamydia pneumoniae-infected lung 3 days after infection (a) shows an interstitial infiltrate consisting predominantly of granulocytes. After 3 days, inflammatory infiltrates within the interstitium, the bronchiolar and alveolar space decreased and became dominated by mononuclear cells (b, 7 days post-infection). $C$. pneumoniae antigen was detected in both cell types by immunohistochemistry (c, granulocytes, 3 days post-infection; d, macrophages, 7 days post-infection, genus-specific antibody CF-2), and in bronchiolar epithelium (e, 7 days post-infection) and alveolar epithelium (f, 7 days postinfection). C. pneumoniae antigen was detected in peribronchiolar lymphatic tissue 7 days postinfection (g). Infected cells were identified as macrophages by a specific antibody RAM 11 in a consecutive section (h). Chlamydial antigen was detected in the spleen (i) and in the media of the aorta, where spindle-shaped cells resembling smooth muscle cells were infected (j). Scale bar $=100 \mu \mathrm{m}$. 
Table 1.-Detection (+) of Chlamydia pneumoniae by immunohistochemistry (IHC) and polymerase chain reaction (PCR) in various organs after single and triple intratracheal inoculation of New Zealand White rabbits

$\frac{\text { Lung }}{\text { IHC PCR }} \frac{\text { PBMC }}{\text { PCR }} \frac{\text { Spleen }}{\text { IHC PCR }} \frac{\text { Aorta }}{\text { IHC PCR }}$

\begin{tabular}{llllllll}
\hline $\begin{array}{l}\text { Single inoculation } \\
12 \mathrm{~h}\end{array}$ & & & & & & & \\
1 day & $+/+^{*}$ & $+/+$ & $+/+$ & $-/-$ & $-/-$ & $-/-$ & $+/+$ \\
3 days & $+/+$ & $+/+$ & $-/+$ & $+/-$ & $+/-$ & $-/-$ & $-/+$ \\
7 days & $+/+$ & $+/+$ & $-/-$ & $-/-$ & $-/-$ & $-/-$ & $+/+$ \\
2 weeks & $+/+$ & $+/+$ & $+/+$ & $-/-$ & $-/-$ & $-/-$ & $+/+$ \\
8 weeks & $-/-$ & $+/+$ & $+/+$ & $-/+$ & $-/-$ & $+/-$ & $+/+$ \\
Triple inoculations & $-/-$ & $+/-$ & $-/-$ & $-/-$ & $-/-$ & $-/-$ & $-/-$ \\
2 weeks & & & & & & & \\
4 weeks & $+/+$ & $+/+$ & $+/-$ & $+/-$ & $-/-$ & $-/-$ & $+/-$ \\
8 weeks & $-/-$ & $-/-$ & $-/-$ & $-/-$ & $-/-$ & $-/-$ & $+/-$ \\
\hline
\end{tabular}

PBMC: peripheral blood monocytes. \#: two animals at each time point (animal 1/animal 2); ${ }^{\text {: }}$ time after last of three inoculations (days $0,4,7$ ).

by PCR, chlamydial DNA was present for up to 2 weeks. Samples of the spleen were occasionally positive for C. pneumoniae by PCR or IHC (fig. 1i). There was a poor correlation between the two methods. Chlamydial DNA was detected in the aorta for up to 4 weeks. Chlamydial antigen was demonstrated by IHC in only one sample 2 weeks postinfection (fig. 1j).

\section{In vitro infection of granulocytes}

Immunofluorescence staining for C. pneumoniae of in-vitroinfected rabbit granulocytes showed intracellular presence of C. pneumoniae LPS 4 days after inoculation (fig. 2a). The number of infected cells increased from $\sim 20 \%$ after 2 days to $\sim 90 \%$ after 4 days, as uninfected cells underwent apoptosis. Infection with $C$. pneumoniae delayed apoptosis of granulocytes (data not shown). Chlamydial viability was proven by subcultivation on HEp-2 cells. Typical inclusions were then detected in $\sim 5 \%$ of the HEp- 2 cells in the second passage, proving retained chlamydial viability after a granulocyte passage (fig. 2b).

\section{Discussion}

In this study, the pulmonary infection and systemic dissemination of $C$. pneumoniae are described in a rabbit model. Its presence in the lungs, lung-associated lymphatic tissue, the spleen and the vasculature were studied, and the cell types involved in the course of infection were identified.

The pulmonary manifestation was histologically characterised by interstitial, intra-alveolar and intrabronchiolar infiltrates of granulocytes and mononuclear cells. It could be divided into an early and a late phase: during the first 3 days of infection, granulocytes dominated the number of macrophages; the later course was characterised by heavy infiltrates of macrophages, while only a few granulocytes were present. C. pneumoniae antigen was present in both cell types. The authors of this study wanted to establish whether the chlamydial antigen in granulocytes represented living organisms and, hence, it was shown for the first time that C. pneumoniae can infect and survive within granulocytes, as shown by cultural recovery from infected granulocytes in vitro. This suggests that granulocytes are involved in the dissemination of the infection and that the granulocyte-mediated
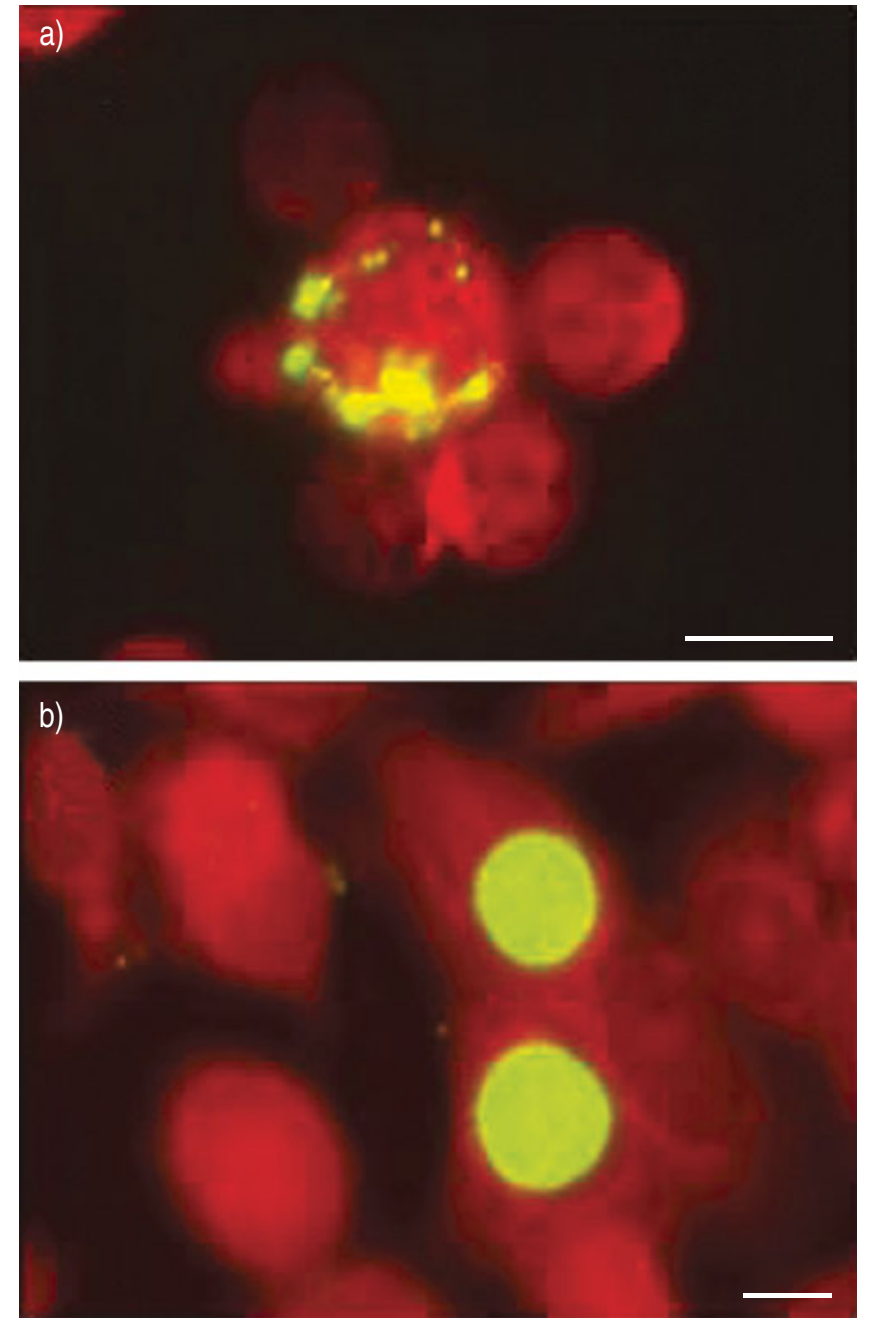

Fig. 2.-Immunofluorescence stain of Chlamydia pneumoniae-(antilipopolysaccharide monoclonal anitbody) infected rabbit granulocytes 2 days after infection in vitro (a). Viability was proven by passing infected granulocytes on HEp-2 cells and immunofluorescence staining of the second passage (b). Scale bar $=10 \mu \mathrm{m}$.

antimicrobial effector mechanisms are not able to prevent establishment of pulmonary infection but rather promote it. Granulocytes do not re-enter the systemic circulation and have a short life span, however, they can transmit the infection to epithelial cells or AM, which are host cells for C. pneumoniae [13] and are able to phagocytose infected granulocytes. Moreover, AM have been shown to migrate through the mucosal barrier [14]. Apparently, granulocytes can act as a kind of Trojan horse for $C$. pneumoniae in the early stage of infection, granting access to the AM, which arrive later and can disseminate the pathogen. Accordingly, C. pneumoniae-infected macrophages were detected in the lung-associated lymphatic tissue, which suggests that the infection of macrophages is the mechanism by which $C$. pneumoniae bypass the mucosal barrier and gain access to the systemic circulation. This idea is supported by the detection of C. pneumoniae DNA in the majority of the PBMC samples. Experiments by others have confirmed the role of macrophages as vector cell. Macrophages were adoptively transferred by intraperitoneal injection from infected to uninfected mice, and C. pneumoniae DNA was later detected in the lungs, thymus, spleen or abdominal lymph nodes [9]. However, primary intraperitoneal infection does not occur naturally. 
In this study, the dissemination into the spleen was studied and the pathogen was detected in low numbers and frequency. As the morphology of the infected cells resembles macrophages, the current authors speculate that the macrophages that acquired the infection in the lungs migrated through the lymphatic system into the circulation and finally got sequestered into the spleen. C. pneumoniae DNA was further detected in the majority of aortic samples and antigen was also detected by IHC in one sample, where few cells of the media harboured the pathogen. The cell type could not be determined but morphology was consistent with a phagocyte or a smooth muscle cell, cell types that have previously been shown to act as target cells in the vasculature [15]. Here, PCR was clearly more sensitive than IHC. The result again substantiates the idea of monocytes/macrophages acting as a vector system for the systemic spread of $C$. pneumoniae.

C. pneumoniae antigen and genomic DNA were tested for in parallel. PCR was more sensitive in comparison to IHC, as only two spleen samples positive by $\mathrm{ICH}$ were negative by PCR. Triple infection did not result in a significantly more severe or longer course of infection, a single intatracheal inoculation was sufficient to establish infection for up to 8 weeks.

It is remarkable that other pathogens like Bacillus anthracis use a similar strategy to invade the systemic circulation. The bacteria infect AM, germinate and escape from the phagosome into the cytosol [16]. They are carried through the mucosal barrier by the host cell. Due to a reduced viability of infected macrophages, the bacteria emerge into the bloodstream, multiply and cause the characteristics of an anthrax infection. C. pneumoniae elementary bodies have never been detected circulating freely in the bloodstream. Thus, the pathogen depends on a host cell to reach an atherosclerotic plaque and to infect the vascular wall. It is not know whether the same infected macrophage that migrated from the lungs into the blood stream is able to further invade an atherosclerotic plaque. The macrophages might be trapped in the spleen where $C$. pneumoniae can infect other blood cells like monocytes or lymphocytes. These cells have been shown to be infected with $C$. pneumoniae in vivo and are able to invade an atherosclerotic plaque [17-19].

Rabbit models for Chlamydia pneumoniae infection have been described previously $[20,21]$. The authors of the present study focused on the cell types involved in the infection and dissemination. While granulocytes have been described in cell infiltrates of Chlamydia pneumoniae-infected animals before [21], it has been shown here for the first time that they are target cells for Chlamydia pneumoniae in vivo and that they can be productively infected in vitro. The importance of AMs for the spread of Chlamydia pneumoniae into the lymphatic tissue and the systemic circulation has been further demonstrated, showing that a vascular infection can result from a respiratory challenge with the pathogen.

Acknowledgements. The authors would like to thank H. Brade (Research Center Borstel, Borstel, Germany) for the anti-chlamydia LPS antibody.

\section{References}

1. Grayston JT. Infections caused by Chlamydia pneumoniae strain TWAR. Clin Infect Dis 1992; 15: 757-761.

2. Saikku P. Epidemiology of Chlamydia pneumoniae in atherosclerosis. Am Heart $J$ 1999; 138: S500-S503.

3. Gieffers J, Pohl D, Treib J, et al. Presence of Chlamydia pneumoniae DNA in the cerebral spinal fluid is a common phenomenon in a variety of neurological diseases and not restricted to multiple sclerosis. Ann Neurol 2001; 49: 585589.

4. Balin BJ, Gerard HC, Arking EJ, et al. Identification and localization of Chlamydia pneumoniae in Alzheimer's brain. Med Microbiol Immunol 1998; 187: 23-42.

5. Grayston JT, Campbell LA. The role of Chlamydia pneumoniae in atherosclerosis. Clin Infect Dis 1999; 28: 993-994.

6. Boman J, Soderberg S, Forsberg J, et al. High prevalence of Chlamydia pneumoniae DNA in peripheral blood mononuclear cells in patients with cardiovascular disease and in middle-aged blood donors. J Infect Dis 1998; 178: 274-277.

7. Gieffers J, Fullgraf H, Jahn J, et al. Chlamydia pneumoniae infection in circulating human monocytes is refractory to antibiotic treatment. Circulation 2001; 103: 351-356.

8. Gaydos CA, Summersgill JT, Sahney NN, Ramirez JA, Quinn TC. Replication of Chlamydia pneumoniae in vitro in human macrophages, endothelial cells, and aortic artery smooth muscle cells. Infect Immun 1996; 64: 1614-1620.

9. Moazed TC, Kuo CC, Grayston JT, Campbell LA. Evidence of systemic dissemination of Chlamydia pneumoniae via macrophages in the mouse. J Infect Dis 1998; 177: 13221325.

10. Maass M, Bartels C, Engel PM, Mamat U, Sievers HH. Endovascular presence of viable Chlamydia pneumoniae is a common phenomenon in coronary artery disease. $\mathrm{J}$ Am Coll Cardiol 1998; 31: 827-832.

11. Dowell SF, Peeling RW, Boman J, et al. Standardizing Chlamydia pneumoniae assays: recommendations from the Centers for Disease Control and Prevention (USA) and the Laboratory Centre for Disease Control (Canada). Clin Infect Dis 2001; 33: 492-503.

12. Maass M, Bartels C, Kruger S, Krause E, Engel PM, Dalhoff $\mathrm{K}$. Endovascular presence of Chlamydia pneumoniae DNA is a generalized phenomenon in atherosclerotic vascular disease. Atherosclerosis 1998; 140: S25-S30.

13. Redecke V, Dalhoff $\mathrm{K}$, Bohnet S, Braun J, Maass $\mathrm{M}$. Interaction of Chlamydia pneumoniae and human alveolar macrophages: infection and inflammatory response. $\mathrm{Am}$ J Respir Cell Mol Biol 1998; 19: 721-727.

14. Harmsen AG, Muggenburg BA, Snipes MB, Bice DE. The role of macrophages in particle translocation from lungs to lymph nodes. Science 1985; 230: 1277-1280.

15. Davidson M, Kuo CC, Middaugh JP, et al. Confirmed previous infection with Chlamydia pneumoniae (TWAR) and its presence in early coronary atherosclerosis. Circulation 1998; 98: 628-633.

16. Guidi Rontani C. The alveolar macrophage: the Trojan horse of Bacillus anthracis. Trends Microbiol 2002; 10: 405409.

17. Kaul R, Uphoff J, Wiedeman J, Yadlapalli S, Wenman WM. Detection of Chlamydia pneumoniae DNA in CD3+ lymphocytes from healthy blood donors and patients with coronary artery disease. Circulation 2000; 102: 2341-2346.

18. Heinemann M, Susa M, Simnacher U, Marre R, Essig A. Growth of Chlamydia pneumoniae induces cytokine production and expression of CD14 in a human monocytic cell line. Infect Immun 1996; 64: 4872-4875.

19. Kaukoranta-Tolvanen SS, Teppo AM, Laitinen K, Saikku $\mathrm{P}$, Linnavuori K, Leinonen M. Growth of Chlamydia pneumoniae in cultured human peripheral blood mononuclear cells and induction of a cytokine response. Microb Pathog 1996; 21: 215-221.

20. Fong IW, Chiu B, Viira E, Fong MW, Jang D, Mahony J. Rabbit model for Chlamydia pneumoniae infection. J Clin Microbiol 1997; 35: 48-52.

21. Moazed TC, Kuo C, Patton DL, Grayston JT, Campbell LA. Experimental rabbit models of Chlamydia pneumoniae infection. Am J Pathol 1996; 148: 667-676. 